Examination of the pus shows the presence of a number of grey opaque points, just visible to the naked eye, which when transferred to a slide and examined under a one-inch objective are seen to be colonies of the botryomyces equi.

They are easily examined in a drop of water or glycerine, but come out beautifully when stained with rubin and orange.

Of half-a-dozen colonies measured, the size varied from $0.2 \mathrm{~mm}$, to $0.45 \mathrm{~mm}$.

\title{
INTRACEREBRAL INJECTION OF ANTITETANIN FOR THE CURE OF TETANUS IN THE HORSEx
}

\author{
By Sidney Vili.ar, F.R.C.V.S., Harrow.
}

CASE I.-On 9th June of the present year a small black male pony was brought to my Infirmary affected with tetanus. The animal was distressed somewhat by its walk of 3 miles, the disease was in its initial stage, and, although the usual symptoms were well marked, it quickly got over the effects of the journey.

The course of the disease in this case was subacute. The pony was put in a loose box, kept perfectly quiet, and had solution of extract of belladonna daily in its drinking water, being mean while well nursed and supplied with suitable food.

It continued to get gradually worse, and, as on the $7^{\text {th }}$ June trismus was becoming very marked, I determined to resort to the intracerebral injection of pure antitetanin ${ }^{1}$, the pony being a suitable subject for this operation on account of its small size and gentle disposition.

On the morning of I 8 th June, having well washed the skin of the forehead with perchloride of mercury soap, shaved it as closely as the movements of the pony would permit, and having injected subcutaneously 3 grains of cocaine in solution, I incised and dissected an angular flap of skin about one-and-a-half inches in length, and cut through the temporal muscles and for a small space detached them from the bone beneath, thus exposing a small portion of the right parietal bone at a distance of one inch from the parietal ridge, on a line with the anterior margin of the root of the ear. With an archimedian drill, bought at an ironmongers for Is. $6 \mathrm{~d}$., a small circular hole was made through the bone, the length of the drill being previously limited to rather more than an inch by threading a cork on it. Through the hole thus made the needle of an ordinary hypodermic syringe was pushed right into the substance of the cerebral hemisphere, and about $3 \mathrm{cc}$. of pure antitetanin was injected.

The needle having been withdrawn, the wound was dressed with iodoform and cotton wool.

Throughout the operation the pony gave no indication of experiencing inconvenience; it was returned to the box and not seen by me again for twenty-four hours, at the end of which period there was a very marked abatement of the tetanic symptoms. The groom said it had emptied its manger during the night, and it was feeding fairly well when I saw it. During the next ten days the tetanic symptoms

1 See article by MM. E. Roux and A. Borrel, "Journal of "Comparative Pathology and Therapeutics," June 1898, Vol. XI., Part II. 
gradually disappeared, and at the end of that time it was turned out into a paddock, having made a good recovery.

The wound in the forchead was not touched until the third day after operating, when, as it was suppurating, it was cleansed and dressed with iodoform. This was repeated when necessary, and when the pony passed from my observation on 7 th August only a small scar indicated the seat of the operation.

Throughout there was no indication of any ill effect from injecting into the brain substance. The animal was a trick pony belonging to a travelling circus, and it had not forgotten its performance, so that its mental capacity did not appear to be affected.

CASE II.-On the 2nd November last a hunter received a punctured wound in the off hind heel, and on the 23 rd of the same month tetanus supervened. The course of the disease was acute, and by the 26 th the tetanic spasms were very severe, and trismus was very marked. On the morning of $26 \mathrm{th}$, having injected 6 grains of cocaine under the skin, a hole was drilled in the parietal bone in the same way as in the case of the pony, and with my ordinary tuberculin syringe I slowly injected $8 \mathrm{cc}$. of pure antitetanin, pushing the needle into the cranial cavity to a depth of 2 inches.

Immediately after the withdrawal of the needle the horse became very violent, its limbs were convulsed, defecation occurred, and it appeared to be about to fall as though in an epileptic fit. The horse, however, supported himself against the wall, and within five minutes the severity of the convulsions had passed off, but it was impossible to dress the wound on his forehead except by throwing a little iodoform on it.

On the morning of 27 th the symptoms of tetanus had considerably lessened, there being, as in the previous case, a very great improvement; the horse was feeding well, and the movements of both limbs and jaws were much less stiff.

The recovery has since been gradual, without any ill effect from the injection; the horse has gone to walking exercise daily since 8th December, and to-day (I 2 th December) he is practically well.

He has always objected to the wound on his forehead being dressed, so that it has had very little attention; nevertheless, it has done well, and now only a small scab remains.

My experience has been that recovery from an attack of tetanus in the horse usually takes six weeks, but in both these cases the duration was scarcely three weeks, although in one a delay of nine days, and in the other of three days, was permitted before resorting to the injecttion.

These are the only cases in which I have made use of the intracerebral injection of antitetanin; the results are so encouraging that perhaps other veterinary surgeons will give this treatment a trial.

In some future case I propose injecting both cerebral hemispheres at an interval of twenty-four hours, anticipating that recovery will be more rapid than when a single injection only is made.

The antitetanin was kindly supplied to me by Dr George Dean of the Jenner Institute of Preventive Medicine. It was quite fresh and pure, Dr Dean being of opinion that an admixture of even a small quantity of carbolic acid would when injected into the brain produce intoxication. 